\title{
The use of underactuation in prosthetic grasping
}

\author{
P. J. Kyberd, A. Clawson, and B. Jones \\ Institute of Biomedical Engineering, University of New Brunswick, Fredericton, \\ New Brunswick, E3B 4A3, Canada
}

Received: 6 March 2010 - Revised: 31 July 2010 - Accepted: 19 August 2010 - Published: 8 February 2011

\begin{abstract}
Underactuation as a method of driving prosthetic hands has a long history. The pragmatic requirements of such a device to be light enough to be worn and used regularly have meant that any multi degree of freedom prosthetic hand must have fewer actuators than the usable degrees of freedom. Aesthetics ensures that while the hand needs five fingers, five actuators have considerable mass, and only in recent years has it even been possible to construct a practical anthropomorphic hand with five motors. Thus there is an important trade off as to which fingers are driven, and which joints on which fingers are actuated, and how the forces are distributed to create a functional device. This paper outlines some of the historical solutions created for this problem and includes those designs of recent years that are now beginning to be used in the commercial environment.
\end{abstract}

This paper was presented at the IFToMM/ASME International Workshop on

Underactuated Grasping (UG2010), 19 August 2010, Montréal, Canada.

\section{Introduction}

The loss of a hand can be a significant impairment to any individual. In a society where independence and self determination is important, the inability to perform two handed tasks can reduce a person's capacity to function easily in daily life. This alone is sufficient motivation to create effective prosthetic hands to replace the loss. However, while the adaptability of homo sapiens means that a loss of a single hand below the elbow can be less a handicap than an inconvenience, it is the methods by which the person compensates for the loss than have a significant impact on their lives. To place a prosthetic hand, or the end of a remnant limb, in the correct place to manipulate an object a person may have to, flex, bend or stretch further than they would if they did not have the loss (Zinck, 2008). With these poorer biomechanics the person may have to use greater forces and larger ranges of motions, these actions are associated with overuse injuries (Kidd et al., 2000) which are observed in long term users of prosthetic arms (Jones and Davidson, 1999) and this ultimately may disable the person more comprehensively. Thus the design of an effective prosthetic hand is an important challenge for engineers, prosthetists and therapists.

The requirement that the prosthesis can be derived from questionnaires of the general population (Atkins et al., 1996; Fraser, 1998; Heger et al., 1985; Kyberd et al., 2007) among others. What is observed it that it must be a practical device. This creates some severe constraints on the design: It has to be light enough to be worn all day, easy to control, cheap enough to be readily available, robust enough to operate in the field for months at a time without the need for servicing. Additionally, it must take high levels of abuse.

While the natural hand has its drives in the forearm, for a prosthesis this is impractical. The level at which the loss occurs is unique to the individual, and the numbers of persons with a particular level of loss increase the further down the arm (Kyberd et al., 1998a). So that any design that uses any part of the forearm for drives of the hand limits its potential market considerably. Any device constructed in this way is not a prosthesis (despite any claims to the contrary), but an anthropomorphic robot, and of little clinical use or significance. 
The result of these requirements is that the number of actuators is limited to the space in the hand and by the mass of the motors. Hence there is a need to gain function from limited actuation, as a result, the use of underactuation in prosthetics has long been considered a solution (Borchardt et al., 1919). Recent years have seen a renewed interest in multi-articulated prosthetic hands and rise in the complexity of commercial devices. This paper outlines some of the solutions that have been suggested in the past and relates them to designs that are being proposed currently. It starts with a review of the constraints on the design.

\section{Solutions}

\subsection{Appearance and the choice of the number of digits}

While appearance is important to some users, it is not essential (Kyberd et al., 1998a), but the majority of effort towards multifunctional terminal devices has been towards fully anthropomorphic hands. This means that the device will possess five digits, but the choice on how they are driven will dictate how functionally effective the device is.

The principle role of the prosthetic hand is to hold objects stably, and the aim of a complex hand is to be more functionally capable. The hand must therefore adapt to a wide range of target objects of different size, shape, mass and surface finishes. Additionally the world it is used is the one that was developed by human beings, so the majority of objects have been made to a particular size, shape and mass to be easily manipulated by the human hand. Thus even an ideal prosthesis shape, without any reference to appearance, may well be similar to a natural hand.

The natural hand achieves its breadth of capability through the use of multiple fingers which are loosely coupled together. The fingers are able to adapt their posture in response to the way the held object is going to be used/carried. To keep the physical effort to a minimum, the hand conforms round the object and spreads the grip forces across as large an area as possible, and so the grip force required is as low as practical (Schulz et al., 2005).

\subsection{Flexible actuation}

While cables create a flexible drive that will fit round corners and allow the actuators to be distant from the motion, it remains difficult to create robust solutions with cables. Strong termination is complex, cables tend to stretch and require constant maintenance. So, for example, although the MIT/Utah dextrous hand (Jaconsen et al., 1986) was derived from a prosthetics research program (Sears et al., 1988), its use of cables meant that it was in constant need of servicing and adjusting, which would preclude it from a prosthetic solution. Instead rigid links are generally used. The flexibility is then created using some form of differential. One solution is to use links in the form of a whiffle tree. All elements are moved by a single drive, when one link is stopped the others continue to move until they all are halted. A similar process can be achieved using a geared differential, where the relative proportions are those of the gear ratios (see Sect. 3). The biggest disadvantage to this form of actuation is to easily return of the system to its start point. While motion in one direction (the "forward" direction) is balanced between the different branches, all elements move together, but the reverse direction tends to channel all the motion from the drive through the branch that is easiest to move. Once it has reached the end the second easiest will move and so on. Thus for a hand, instead of all the fingers extending together, one moves quickly to fully extended, before the second, etcetera. In order to balance the branches on the return leg, an external return force is required, such as springs, which would add mass and complexity to the hand. The spring also opposes the flexion drive, hence weakening the grip.

\subsection{Digit selection}

While there are theoretical considerations as to the minimum number of digits necessary to stably hold an abstract object, other matters dominate with a prosthesis. For example; the requirement that the device is natural in appearance ensures that there are five curling fingers. Effective manipulation can be conducted with three digits, but a fourth and fifth increases the chances of a stable contact. When a hand is used in an unstructured environment this level of redundancy and flexibility is important. Having to adjust the grip or change the posture to get sufficient contact is not readily tolerated by prosthesis users.

\subsubsection{Classes of grip}

With many fingers different grip forms can be created. The hand has a single opposing digit (the thumb) and most grips are controlled by its use. There are numerous different ways to classify grasp types. Napier (1956) suggested two: Precision (where the tips of the fingers oppose each other) and Power (where the fingers thumb wrap around the object and the hand is able to impart more force). Others have seen six broad grasps (Kamakura et al., 1980; MacKenzie and Iberall, 1994) (tripod, cylinder, extension, tip, lateral and spherical). While these summarise the grip shapes the hand must perform, Iberall simplified the matter, considering only what part of the hand the tips of the fingers oppose on to: So Precision grips are where the finger tips opposed the thumb tip, Power-like grips, when the finger tips oppose the palm, or Lateral grips, when the thumb tip opposes the side of the flexed index finger (Iberall, 1997). This final form of classification gives the engineer clear indications as to the required path the digit tips must move through to create as wider a range of grips (and so function) for the hand. To achieve all these grasps the fingers need to be driven separately to the thumb and they need to curl to reach the palm. A prosthesis 
should be able to perform all of these grips to be truly practical. When compromises are made it might be imagined that the most common grips are those which are considered first. However, the differences in the way that a prosthesis is used, compared with a natural hand means that it is still uncertain which grips are most useful. The latest generation of commercial hands use the lateral grip more often than the natural hand does, (proportions according to Sollerman and Sperling (1978)) and also for gripping objects not considered for this grip in natural prehension.

\subsubsection{Precision grips}

An important distinction are the two and three jaw precision grips (tripod and tip). Two jaw opposition dictates which fingers are linked to the same drive. Two general solutions have been investigated; each splits the fingers into two groups: Either two fingers in each group or three in one group and the index driven separately. Early examples of these two solutions are the Belgrade Hand (Bekey et al., 1990; Iberall et al., 1996) and the Southampton Hand (Todd, 1969; Kyberd et al., 2001). The latter split is similar to the way work gloves are designed for use in cold climates, there the three fingers are set in a mitt for warmth and the index is kept separate for ease of manipulation. In both of these hands the drives passed through whiffle trees to the fingers, with two motors in the palm for four fingers. Both formats allow balancing of forces across the surface of the held object with the grasp curving round the outside of an object.

Both also allowed for two point grips between the index finger and the thumb. In the case of the Belgrade Hand the middle finger needs to close fully once the thumb was opposed by the index, to allow the index to grip the object. With the Southampton Hand the three fingers could be held in the palm (or extended out of the way) and a two point grip maintained (Moore, 1980). A second advantage is that repeated grips with the Belgrade format force both of the fingers to fully open and close again, while with the Southampton Hand the index finger is fully isolated, so the index only needs be opened to reapply the grip.

A third hand with whiffle trees across the palm are the Sven hand series (Almström, 1977). All four fingers were driven through a single whiffle tree, providing grip flexibility from one motor. Later this gave rise to the ES Hand (Almström et al., 1981), which is the only underactutated prosthetic hand to be used in the field (albeit in limited numbers).

\subsection{Thumb actuation}

In multifunction prosthetic hands the role of the thumb can vary considerably. The natural thumb can move in and out from the fingers (flex and extend) as well as across the hand (abduct and adduct). If the thumb can abduct a small amount it can provide both two and three jaw opposition, if it is allowed to move round to the side then the lateral grip is also possible. Some hands require two motors to provide this extra motion (Kyberd, 1990) and the Otto Bock Michelangelo hand. Others, such as the TouchBionics and RSL Steeper hands require external forces from the user to move the thumb across the palm (Newman, 2008). A more sophisticated design is to use opposite ends of a more complex range of motion of the thumb to create motion in one plane or the other (Clawson et al., 2010), or Pons et al. (2005) who used a Geneva mechanism. Thus getting the two motions from a single actuator.

Additionally, in the natural hand the motion of fingers and thumb are different (Wing et al., 1986), with the thumb moving more slowly and allowing the fingers to close on to the thumb, which acts as a reference for the person grasping. So powerful is this need, that in simple prostheses where both fingers and thumb are linked and move with the same angular velocity, users move their forearm in such a way that the relative motions of finger and thumb are closer to the natural response (Wing and Fraser, 1987). Using this knowledge it is clear that if the speed of the fingers and thumb are under the control of the designer, that it is appropriate to allow the thumb to move more slowly to get closer to the natural solution. This also allows the thumb to be geared differently so it can create the power in the grip, in a similar manner to the natural solution (Kyberd et al., 1998b). Most conventional prosthetic hands have the same angular velocity for fingers and thumb.

\subsection{Finger construction}

The natural finger has three joints outside the palm. This allows the finger to wrap around an object and spread the grip across a larger surface area (reducing the need for a greater grip force for a stable grip). The tip of the finger can then oppose the palm at the base of the finger, allowing power grip on a small object. Each natural segment consists of a phalangial bone, linked by a rolling, sliding joint. In a prosthetic replacement uses a link between successive phalanges to give a similar motion of the tip of the finger, Fig. 1. This increase in complexity from rigid fingers to three separate elements, may not provide sufficient functional increase to justify the increase in complexity, for example; The Montreal Hand (Vinet et al., 1995) chose to fix the top two phalanxes in slight flexion and altered the ratios of the lengths of the phalanxes from roughly equal, in the anthropomorphic case, to the distal pair being roughly the same length as the proximal segment, so that with two elements the tips of the fingers still opposed the base of the finger. There is as yet no evidence that this compromise effects the functional capabilities.

Alternatively increased grip flexibility can be achieved if the top joint and the lower joints are linked via a whiffle tree across the finger. In this case the tips of the fingers will continue to close onto an object even when the base of the finger is in contact with the object. This means that the contact area is spread more widely (Kyberd et al., 2001), Fig. 2. 


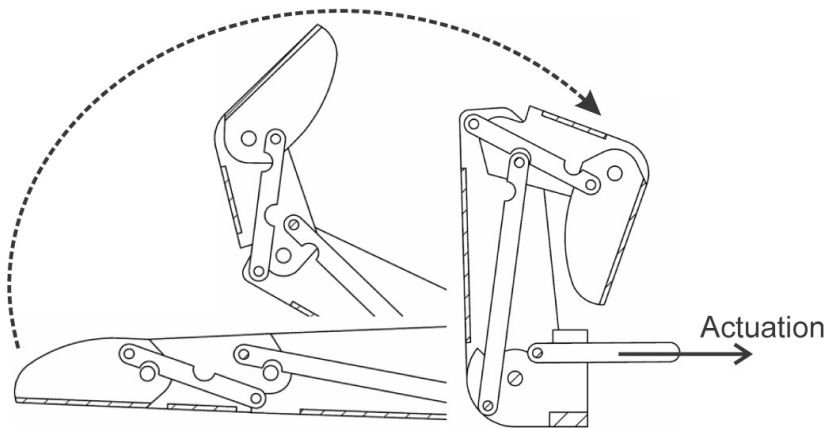

Figure 1. Links between the interphalangial joints cause the finger to flex as it closes. the finger closes progressively, but if the lower phalanx is halted by an external object the tips cannot continue to move.
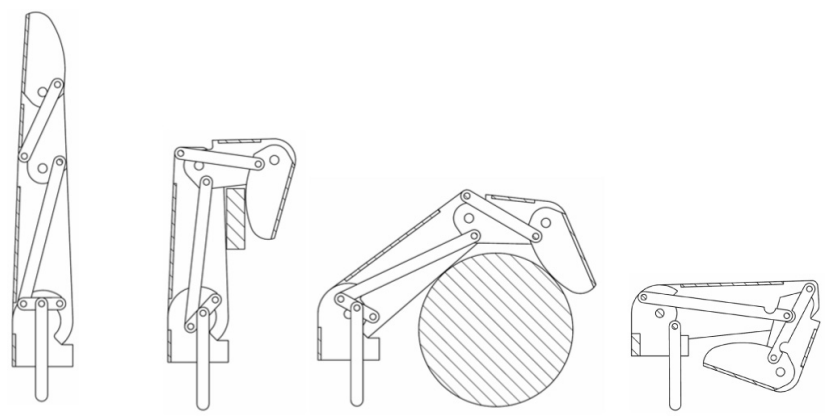

Figure 2. Use of the equalising mechanism across the finger to allow an adaptive grip. if the proximal phalanx is stopped by an external object. the distal phalanx closes onto the object. for a larger object the finger curls similar to the design in Fig. 1.if unobstructed, the tip wraps round to the base of the finger.

\subsection{Power sources}

The overwhelming choice for power sources has been the permanent magnet electric motor. This is again for practical reasons. For example; batteries are easy to recharge in any domestic situation, while the accumulators or pressure vessels needed for pneumatic and hydraulic systems require considerable specialist equipment which is unlikely to be available to the average user, making the maintenance of a device using such sources very much more difficult. Indeed this advantage has increased over the past 20 years as the technology has continued to advance, with increasing capacity and peak power output for simple motors, so that alternative systems remain less practical. One drawback with motors is that they are most efficient when they are moving at a constant rate and in one direction of rotation. Biological systems tend to move slowly, cyclicly and start and stop often. Hence a motor is the poorest choice from the perspective of efficiency. One group in Germany (Schulz et al., 2005) has taken a different approach. They generate pressure for a lightweight hydraulic hand using an electric motor turning at a constant rate. The power source can still be a battery, with all the advantages of their ability to be easily recharged, but the actuator is hydraulic, which is better at low speeds and from rest. Each finger can be linked via an equalising mechanism, but from a single source, with the joints flexing along the fingers and across the hand. This device was undergoing clinical assessment (Schulz et al., 2008).

\section{Current designs}

Historically, numerous solutions have been proposed for prosthetic hands. Some have been used in the field, but the limitations on technology has meant that the mass of the designs has restricted their application. With the launch of the TouchBionics Hand in 2005, a new generation of multifunction hands with flexible fingers is beginning to enjoy genuine clinical use. The TouchBionics hand is fully actuated (Newman, 2008). The next device to reach the market, was the RSL Steeper, "BeBionic" hand in spring 2010. This too has separately driven fingers which curl as they flex and a thumb with unpowered abduction. However neither of these hands are underactuated. Yet to reach the market, is the Otto Bock Michaleangelo hand (Puchhammer, 2008). The main flexion power comes from a single brushless motor in the palm, and a combination of a cam at the opposite ends of the motion and a smaller flexion motor for the thumb, allows the hand to adopt tip and lateral prehension. The fingers do not curl, merely flexing from their base and their motions are linked. The hand is statically very anthropomorphic in appearance, but without some independent clinical testing, it is unclear if these changes give it any functional advantages over the more conventional single degree of freedom designs. Further from the market is the Vicent hand from Schutz's team (Schulz, 2010), with individually driven fingers.

The team at the Institute of Biomedical Engineering at the University of New Brunswick have many years of experience with novel means to control prosthetic systems, most recently they have begun to design a hand which will incorporate their knowledge of pattern recognition with the collected ideas concerning hand design to product a lightweight compact anthropomorphic hand. It has a three and one finger split with the three fingers driven through two stages of differential gears. Each finger is jointed at the lower interphalangial joints. The thumb has a single motor and creates lateral or tip pinch at opposite ends of its travel. Each finger is instrumented so that contact points are recorded and the most appropriate grip for the target object can be automatically selected and the grip force applied will be sufficient to hold the object stably, Fig. 3. If the object slips within the grip it will be detected and the hand automatically grips tighter to arrest any slide. Using this design the hand can match all six of the basic grip classes. 


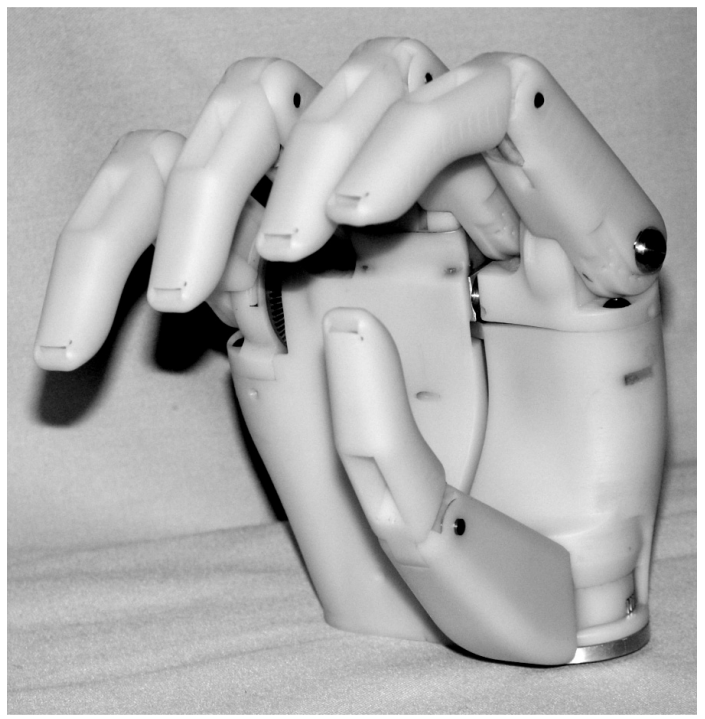

Figure 3. The design of the UNB hand. it incorporates three motors to actuate five fingers, the thumb with two separate motions.

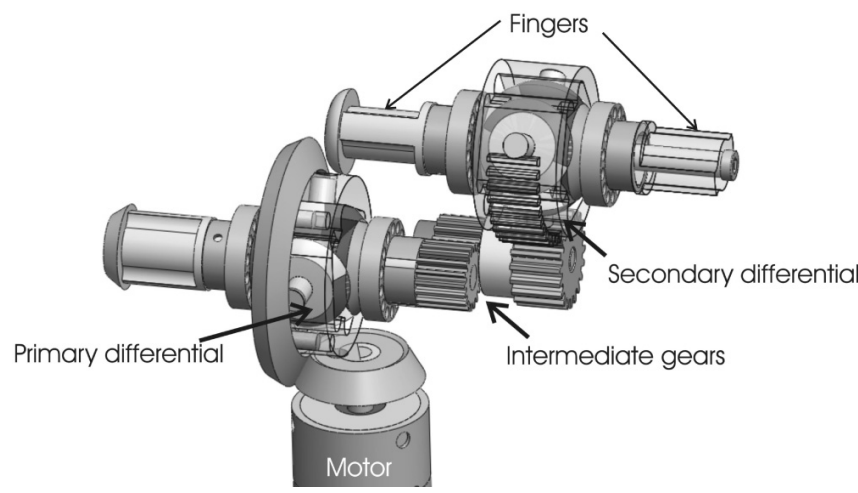

Figure 4. The use of a pair of differentials to create equalising mechanism across two fingers.

The finger drive uses two differentials with three internal crown gears, Fig. 4. The motor drives the housing of the first gear, the output of that gear passes through an intermediate shaft before driving the second differential via spur gearing. This allows there to be a different ratio between the fingers to equalise the forces transmitted. It also means that the base of the fingers is stepped in an anthropomorphic manner. This is different to the the DEKA hand which places the differentials in series and so the bases of the fingers are all in one line (Langefeld et al., 2008).

\section{Conclusions}

The use of underactuation in prosthetics is the result of pragmatic choices to create functional and adaptive hand mechanisms, while reducing size and mass a level that is practical. This has resulted in different numbers of drives and degrees of freedom. To obtain a flexible grip the use of underactuation allows greater flexibility with fewer drives. However few such hands have achieved significant clinical use so far.

Acknowledgements. The authors wish to thank their support, from the Natural Sciences and Engineering Research Council of Canada, the Atlantic Innovation Fund and the New Brunswick Innovation Foundation.

Edited by: J. L. Herder

Reviewed by: two anonymous referees

\section{References}

Almström, C.: An Electronic Control System for a Prosthetic Hand with Six Degrees of Freedom, Tech. Rep. 1:77, Research Laboratory of Medical Electronics, Chalmers University of Technology, 1977.

Almström, C., Herberts, P., and Körner, L.: Experience with Swedish Multifunctional Prosthetic Hands Controlled by Pattern Recognition of Multiple Myoelectric Signals, International Orthopaedics (SICOT), 5, 15-21, 1981.

Atkins, D., Heard, D., and Donovan, D.: Epidemiologic Overview of idividuals with Upper-Limb Loss and their Reported Research Priorities, J. Prosthet. Orthot., 8, 2-11, 1996.

Bekey, G., Tomovic, R., and Zeljkovic, I.: Dextrous Robot Hands, chap. Control Architecture for the Belgrade USC hand, SpringerVerlag, 1990.

Borchardt, Hartmann, Leymann, Radike, Schlesinger, and Schwiening: Ersatzglieder und Arbeitshilfen fr Kriegsbeschädigte und Unfallverletzte, Springer Verglag, 1919.

Clawson, A., Kyberd, P., Jones, B., Losier, Y., Wilson, A., Segil, J., and Weir, R.: The UNB Hand - Multifunction Hand Prosthesis System, in: 13th World Congress of the International Society of Prosthetics and Orthotics, International Society of Prosthetics and Orthotics, 2010.

Fraser, C. M.: Laterality, gender and age differences in estimated frequency and actual registration of people with congenital upper limb absences, Prosthet. Orthot. Int., 22, 224-229, 1998.

Heger, H., Milstien, S., and Hunter, G. A.: Electrically powered prostheses for the adult with an Upper Limb Amputation, J. Bone Joint Surg., 67-B, 278-281, 1985.

Iberall, T.: Human Prehension and Dextrous Robot Hands, Int. J. Robot. Res., 16, 285-299, 1997.

Iberall, T., Sukhatme, G., Beattie, D., and Bekey, G.: Intelligent Control Systems, chap. Control philosophy and simulation of a robotic hand as a model for prosthetic hands, IEEE Press, 682701, 1996.

Jaconsen, S., Iversen, E., Knutti, D., Johnson, R., and Biggers, K.: Design of the Utah/MIT Dexterous Hand, in: IEEE international conferance on robotics and automation, San Francisco, California, IEEE, 1986. 
Jones, L. and Davidson, J.: Save that arm: a study of problems in the remaining arm of unilateral upper limb amputees, Prosthet. Orthot. Int., 23, 55-58, 1999.

Kamakura, N., Matsuo, M., Harumi, I., Mitsuboshi, F., and Miura, Y.: Patterns of static prehension in normal hands, Am. J. Occup. Ther., 34, 437-445, 1980.

Kidd, P., MoCoy, C., and Steenbergen, L.: Repetitive Strain Injuries in Youths, Jo. Am. Acad. Nurse Prac., 12, 413-426, 2000.

Kyberd, P.: The Algorithmic Control of a multifunction hand prosthesis, Ph.D. thesis, Electrical Engineering Department, Southampton University, 1990.

Kyberd, P., Beard, D., Davey, J., and Morrison, D.: Survey of upper limb prostheses users in Oxfordshire, J. Prosthet. Orthot., 10, 8591, 1998a.

Kyberd, P., Evans, M., and te Winkel, S.: An Intelligent Anthropomorphic Hand, with Automatic Grasp, Robotica, 16, 531-536, 1998b.

Kyberd, P., Light, C., Nightlingale, J., Whatley, D., and Evans, M.: The Design of Anthropomorphic Prosthetic Hands, a study of the Southampton Hand, Robotica, 19, 593-600, 2001.

Kyberd, P. J., Wartenberg, C., Sandsjö, L., Jönsson, S., Gow, D., Frid, J., Almström, C., and Sperling, L.: Survey of upper extremity prosthesis users in Sweden and the United Kingdom, J. Prosthet. Orthot., 19, 55-62, 2007.

Langefeld, C., Evans, C., Smith, S., Muller, A., Kerwin, J., Schnellinger, T., Guay, G., and der Merwe, D. V.: Patent number US2008/0288088 - Arm Prosthetic Device, 2008.

MacKenzie, C. and Iberall, T.: The Grasping Hand, North-Holland, 1994.

Moore, D.: Development of multifunctional adaptive hand prosthesis, Ph.D. thesis, Electrical Engineering Department, University of Southampton, 1980.

Napier, J.: The prehensile movements of the human hand, J. Bone Joint Surg., 38B, 902-913, 1956.

Newman, P.: Multi-articulating Hands and Fingers - Technical Strategies for Improved Function and Myoelectric Uptake(Abstract), in: MEC '08 Measuring Success in Upper Limb Prosthetics, UNB, IBME, Fredericton, New Brunswick, Canada, p. 183, 2008.

Pons, J., Ceres, R., Reynaerts, D., Saro, B., Levin, S., and Moorleghem, D. V.: Objectives and technological approach to the development of the multifunctional MANUS upper limb prosthesis, Robotica, 23, 301-310, 2005.
Puchhammer, G.: Michelangelo 03 - A versatile hand prosthesis, featuring superb controllability and sophisticated bio mimicry (Abstract), in: MEC '08 Measuring Success in Upper Limb Prosthetics, UNB, IBME, Fredericton, New Brunswick, Canada, 162-163, 2008.

Schulz, S.: Introducing a New Multiarticulating Myoelectric Hand System, in: 13th World Congress, 10-15 May 2010, Leipzig, Germany, International Society of Prosthetics and Orthotics, ISPO, 598-599, 2010.

Schulz, S., Pylatiuk, C., Reischl, M., Martin, J., and Bretthauer, R. M. G.: A hydraulically driven multifunctional prosthetic hand, Robotica, 23, 293-300, 2005.

Schulz, S., Pylatiuk, C., Kargov, A., Werner, T., Gaiser, I., Reischl, M., and Oberle, R.: Design and Preliminary Experience with Fludihand MK III, in: MEC '08 Measuring Success in Upper Limb Prosthetics, UNB, IBME, Fredericton, New Brunswick, Canada, 197-199, 2008.

Sears, H., Andrew, J., and Jacobsen, S.: Experience with the Utah arm, Hand and Terminal Device, chap. 18, 194-210, SpringerVerlag, 1988.

Sollerman, C. and Sperling, L.: Evaluation of Activities of Daily Living - Especially hand function, Scand. J. Rehabil. Med., 6, 139-143, 1978.

Todd, R.: Adaptive control of a hand prosthesis, Ph.D. thesis, Electrical Engineering Department, University of Southampton, 1969.

Vinet, R., Lozac'h, Y., Beaudry, N., and Drouin, G.: Design methodology for a multifunctional hand prosthesis, J. Rehabil. Res. Dev., 32, 316-324, 1995.

Wing, A. M. and Fraser, C.: The contribution of the thumb to reaching movements, Q. J. Exp. Psychol., 35A, 297-309, 1983.

Wing, A., Turton, A., and Fraser, C.: Grasp size and accuracy of approach in reaching, J. Motor Behav., 18, 245-260, 1986.

Zinck, A.: The Investigation of Compensatory Movements in Prosthesis Users and the Design of a Novel Wrist, Masters, Department of Mechanical Engineering, University of New Brunswick, 2008. 